\title{
Complicações macrovasculares do diabetes melito: peculiaridades clínicas, de diagnóstico e manejo
}

\author{
Macrovascular diabetic complications: clinical \\ characteristics, diagnosis and management
}

Cristina Triches', Beatriz D'Agord Schaan', Jorge Luiz Gross', Mirela Jobim de Azevedo'

1 Programa de Pós-graduação em Ciências Médicas:

Endocrinologia e Metabologia e Serviço de Endocrinologia do Hospital de Clínicas de Porto Alegre, Universidade Federal do Rio Grande do Sul (UFRGS), Porto Alegre, RS, Brasil
Correspondência para: Mirela Jobim de Azevedo Serviço de Endocrinologia do Hospital de Clínicas de Porto Alegre Rua Ramiro Barcelos, 2.350, prédio $12,4^{\circ}$ andar

90035-003 - Porto Alegre, RS, Brasil mirelaazevedo@terra.com.br

Recebido em 13/Abr/2009 Aceito em 29/Jun/2009

\begin{abstract}
RESUMO
O diabetes melito (DM) é um fator de risco independente para doença arterial coronariana, acidente vascular cerebral, doença vascular periférica e insuficiência cardíaca, que são as principais causas de morte nesses pacientes. Além disso, pacientes com DM e doença cardiovascular têm pior prognóstico, por apresentarem menor sobrevida, maior risco de recorrência da doença e pior resposta aos tratamentos propostos. Os avanços diagnósticos e terapêuticos das últimas décadas já mostram uma redução do risco de eventos cardiovasculares nesses pacientes, mas o risco absoluto desses é ainda duas vezes maior em relação ao dos pacientes não diabéticos. Portanto, é prioritária a adoção de um manejo intensivo, com controle rígido dos fatores de risco cardiovasculares. Esta revisão trata das principais características clínicas e apresenta uma abordagem prática do rastreamento, diagnóstico e tratamento da doença macrovascular nos pacientes com DM. Arq Bras Endocrinol Metab. 2009;53(6):699-708

Descritores

Diabetes melito; doença cardiovascular; doença arterial coronariana; fatores de risco; tratamento; diagnóstico
\end{abstract}

\begin{abstract}
Diabetes mellitus (DM) is an independent risk factor for coronary heart disease, stroke, peripheral arterial disease and heart failure, which are the main causes of death in these patients. Moreover, patients with DM and cardiovascular disease have a worse prognosis than nondiabetics, present lower short-term survival, higher risk of recurrence of the disease and a worse response to the treatments proposed. In the last decades, diagnostic and therapeutic progress had already shown benefits concerning cardiovascular risk reduction in these patients, but their absolute mortality risk is still twice that of non-diabetic patients. Because of this, the adoption of intensive treatment, with strict cardiovascular risk factor control, is a priority. The present study presents the main clinical characteristics and also the practical approach for screening, diagnosis and treatment of patients with diabetic macrovascular disease. Arq Bras Endocrinol Metab. 2009;53(6):699-708
\end{abstract}

Keywords

Diabetes mellitus; cardiovascular disease; coronary artery disease; risk factors; treatment; diagnosis

\section{INTRODUÇÃO}

$\Delta$ presença de diabetes melito (DM) é um fator de - risco independente para doença arterial coronariana (DAC), acidente vascular cerebral (AVC), doença vascular periférica (DVP) e insuficiência cardíaca, que são as principais causas de morte nesses pacientes. Estudo transversal que incluiu 927 pacientes com DM tipo 2 (DMT2) atendidos em nível ambulatorial em três centros médicos do Rio Grande do Sul observou prevalência de DAC de 36\%, DVP de 33\% e hipertensão de $73 \%$ (1). O risco relativo para doença cardiovascular (DCV) aumenta de duas a quatro vezes nos pacientes com DMT2 comparados à população em geral. Esses pacientes apresentam frequentemente associação de fa- 
tores de risco cardiovascular: hipertensão arterial sistêmica, valores baixos de HDL séricos, microalbuminúria e doença renal, aumento do índice de massa corpórea (IMC) e da relação cintura quadril $(1,2)$.

A DAC é mais incidente e mais grave nos pacientes com DM em relação a pacientes não diabéticos $(3,4)$. No DM tipo 1 (DMTl), as primeiras manifestações da DAC ocorrem entre a terceira e quarta décadas de vida, principalmente na presença de nefropatia diabética (5). As mulheres com DM são particularmente afetadas pela DAC e seu risco de morte é $50 \%$ maior do que o dos homens com DM. O DM, quando associado a fatores de risco clássicos para DAC, como hipercolesterolemia, fumo e hipertensão arterial sistêmica, aumenta o risco para DCV atribuído a cada um desses fatores de forma mais importante do que é observado em indivíduos sem DM (6).

A doença cerebrovascular tem também maior incidência em pacientes com DM do que em indivíduos não diabéticos. Entre os pacientes com AVC, a prevalência de DM é três vezes maior do que nos indivíduos sem DM (7).

A DVP acomete duas a quatro vezes mais pacientes com DM, atingindo a prevalência de $12 \%$ a $16 \%$ quando avaliada por meio da medida do índice de pressão arterial perna-braço. A presença do DM aumenta o risco de desenvolver isquemia crítica dos membros com DVP. A DVP associada ao DM é a principal causa de amputação não traumática de membros inferiores nos Estados Unidos. Cerca de 50\% das amputações de membros inferiores são relacionadas ao DM e, embora tenha ocorrido uma diminuição importante do risco relativo de amputações nos pacientes com DM quando comparados a indivíduos sem DM, esse risco continua bastante elevado - cerca de oito vezes (8).

\section{CARACTERÍSTICAS CLÍNICAS}

As manifestações clínicas da aterosclerose nos pacientes com DM ocorrem em especial nas artérias coronárias, carótidas, aorta, cerebrais e periféricas (membros inferiores).

A DAC pode apresentar-se com dor anginosa típica ou atípica e, mais frequentemente, é assintomática, ocorrendo em cerca de $55 \%$ dos pacientes com DM (9). Em pacientes com DM, a DAC tem pior prognóstico, com menor sobrevida em curto prazo, maior risco de recorrência e pior resposta aos tratamentos propostos (10). A fatalidade do infarto agudo do miocárdio (IAM) em pacientes diabéticos é duas vezes maior do que em pacientes não diabéticos, em razão da maior frequência de complicações como insuficiência cardíaca, reinfarto e morte súbita (11).

O AVC manifesta-se em pacientes com DM em idade inferior a dos pacientes sem DM, e mulheres com $\mathrm{DM}$ têm maior risco de AVC do que homens com DM (7). A presença de DM piora a evolução dos pacientes com AVC: o risco de recorrência é duplicado, o risco de demência é triplicado e a mortalidade aumenta acentuadamente (12).

Em relação à DVP, embora muitos pacientes tenham redução do índice de pressão arterial perna-braço, poucos apresentam quadro clínico de claudicação intermitente, dor em repouso ou lesão trófica em membros inferiores (13). A duração do DM e a hiperglicemia se associam positivamente à presença e gravidade da DVP. Além do aumento da frequência de DVP, o DM afeta a distribuição da aterosclerose nos membros inferiores, atingindo tipicamente as artérias tibiais, peroneais, femorais e poplíteas. Ainda, as lesões ateroscleróticas nos pacientes diabéticos apresentam calcificações vasculares com maior frequência do que nos pacientes não diabéticos (14).

\section{AVALIAÇÃO DA DOENÇA MACROVASCULAR: DIAGNÓSTICO E ACOMPANHAMENTO}

\section{Avaliação geral}

A tabela 1 resume os principais aspectos sugeridos para a avaliação geral do paciente com DM e macroangiopatia.

$\mathrm{Na}$ avaliação da doença macrovascular, a anamnese deve buscar a presença de sintomas de dor torácica típica ou atípica. Deve-se ter em mente a presença de outros sintomas relacionados à isquemia miocárdica (dispneia, sudorese excessiva, desconforto epigástrico) e que cerca de $50 \%$ dos pacientes com isquemia miocárdica podem ser assintomáticos. A presença de dispneia aos esforços, claudicação intermitente e dor de membros inferiores em repouso (e sua diferenciação da dor neuropática) são sintomas que devem ser questionados diretamente. História de eventos cardiovasculares prévios e familiares precoces (evento cardiovascular no pai antes dos 55 anos e na mãe antes do 65 anos) auxilia na identificação de indivíduos mais predispostos a desenvolverem manifestações clínicas de doença aterosclerótica. O exame físico deve incluir especificamente a ausculta cardíaca, pesquisa de sopros arteriais (carotídeos, abdominais e femorais), palpação de pulsos periféricos e exame cuidadoso dos membros inferiores. A pesquisa de sopro 
carotídeo ajuda a identificar os pacientes com maior risco de DCV e que se beneficiariam de uma estratégia de tratamento mais intensivo. Em uma metanálise recentemente publicada, a presença de sopro carotídeo aumentou cerca de duas vezes o risco de infarto do miocárdio e morte cardiovascular em cinco anos (15).

Recomenda-se a avaliação anual dos fatores de risco cardiovasculares modificáveis: dislipidemia, hipertensão arterial e presença de tabagismo (16). Considerando-se o conceito de risco cardiovascular global, pacientes com DM, mesmo sem doença aterosclerótica estabelecida, apresentam risco elevado de desenvolver eventos vasculares no futuro. Este dado determinou que o DM passasse a ser considerado como um "equivalente de DCV estabelecida" (17). Entretanto, como o risco absoluto para eventos cardiovasculares não é o mesmo em todos os pacientes com DM $(18,19)$, sugere-se a utilização de escores de risco cardiovascular como, por exemplo, o escore de Framingham, que inclui fatores de risco cardiovasculares tradicionais (idade, sexo, fumo, pressão arterial (PA), colesterol total e HDL-colesterol) ou, idealmente, o UKPDS risk engine, que foi validado especificamente para pacientes com DM e inclui também valores de HbAlc, duração de DM, etnia e presença de fibrilação atrial (20).

Fatores de risco não tradicionais podem ser também utilizados na avaliação do risco cardiovascular, porém sua utilidade clínica ainda não está amplamente comprovada. Um fator de risco não tradicional e intensamente estudado é a medida da proteína C-reativa ultrassensível. Em indivíduos com risco cardiovascular intermediário ( $10 \%$ a $20 \%$ de risco para evento cardiovascular em dez anos), é fator de risco adicional para morte por DCV, embora esse aspecto ainda não esteja bem estabelecido em pacientes com DM (21). Recentemente, os resultados do estudo JUPITER mostraram que o emprego de $20 \mathrm{mg}$ de rosuvastatina em cerca de 17 mil pacientes sem DM com valores de proteína Creativa $>2,0 \mathrm{mg} / \mathrm{L}$ e $\mathrm{LDL}<130 \mathrm{mg} / \mathrm{dL}$ determinou uma redução de aproximadamente $45 \%$ no aparecimento de eventos cardiovasculares maiores após 1,9 ano de acompanhamento (22). Deve ser também lembrado que a doença periodontal, mais frequente em pacientes com DM, é também um fator de risco independente para DAC nesses pacientes (23).

A avaliação nutricional, antropométrica e de revisão de hábitos de vida (atividade física, álcool, tabagismo) deve ser realizada regularmente com ênfase à presença de obesidade, sobrepeso e sedentarismo, frequentes no paciente com DMT2, pois seu tratamento implica melhora do controle glicêmico, lipídico e pressórico.

$\mathrm{O}$ controle glicêmico intensivo (alvo HbAlc $<7 \%$ ) está associado a uma redução de complicações cardiovasculares (24-26), embora não se observem efeitos benéficos sobre a DVP. A automonitorização de glicemia capilar está indicada em usuários de insulina. Glicemia de jejum, HbAlc e glicemia pós-prandial (se valores de HbAlc elevados em vigência de glicemia de jejum normais) devem ser avaliados pelo menos a cada quatro meses (16).

A PA deve ser avaliada em todas as consultas de rotina, lembrando que, em pacientes com DM, o controle pressórico intensivo é capaz de reduzir complicações crônicas, desde que mantido $(27,28)$. O diagnóstico de hipertensão é definido por valores de $\mathrm{PA} \geq 140 / 90$ $\mathrm{mmHg}(29)$, embora deva ser enfatizado que, em pa-

\begin{tabular}{|c|c|c|}
\hline Tipo de avaliação & Fatores em avaliação & Frequência de avaliação \\
\hline \multirow[t]{2}{*}{ Geral } & $\begin{array}{l}\text { Anamnese: dor torácica típica ou atípica, dispneia, claudicação intermitente, tempo conhecido } \\
\text { de DM, eventos cardiovasculares e amputações prévias }\end{array}$ & $4 / 4$ meses \\
\hline & $\begin{array}{l}\text { Exame físico: ausculta cardíaca, pesquisa de sopros (carotídeos, abdominais, femorais), exame } \\
\text { de membros inferiores com palpação de pulsos periféricos }\end{array}$ & \\
\hline Controle glicêmico & $\begin{array}{l}\text { HbA1c, glicemia de jejum (glicemia pós-prandial se HbA1c alta e glicemia de jejum normal), } \\
\text { glicemia capilar (usuários de insulina) }\end{array}$ & $4 / 4$ meses \\
\hline Controle pressórico & Medida da pressão arterial supina e após 1 minuto em ortostatismo & $4 / 4$ meses \\
\hline Perfil lipídico & Colesterol total, LDL, HDL e triglicerídeos & Anual \\
\hline Outros fatores de risco modificáveis & Índice de massa corpórea, tabagismo, atividade física e dieta & 4/4 meses \\
\hline Cálculo do risco cardiovascular & UKPDS risk engine: http://www.dtu.ox.ac.uk/index.php?maindoc=/riskengine/ & Anual \\
\hline Avaliação renal & $\begin{array}{l}\text { Albuminúria em amostra, taxa de filtração glomerular estimada } \\
\text { (MDRD: http://www.kidney.org/klsprofessionals/gfr_calculator.cfm) }\end{array}$ & Anual \\
\hline Outras & $\begin{array}{l}\text { Ecografia de carótidas } \\
\text { EcoDoppler/ressonância magnética com angiografia de artérias renais } \\
\text { Pesquisa de cálcio coronário }\end{array}$ & Individualizar \\
\hline
\end{tabular}


cientes com DM, o alvo pressórico é uma $\mathrm{PA} \leq 130 / 80$ mmHg (16). A PA deve ser medida na posição supina e em ortostatismo, pois a hipotensão postural (redução $\geq 20 \mathrm{mmHg}$ na PA sistólica da posição supina para o ortostatismo, após um minuto) pode ser um dos sinais clínicos de neuropatia autonômica.

A monitorização ambulatorial da pressão arterial (MAPA) em 24 horas permite a avaliação do tratamento anti-hipertensivo (29). Também faz o diagnóstico de "hipertensão do avental branco" (PA 24 horas normal na MAPA e elevada no consultório) e "hipertensão mascarada" (PA 24 horas elevada na MAPA e normal no consultório), que ocorrem em 14,4\% (30) e 30\% (31) dos pacientes com DM, respectivamente. A "hipertensão do avental branco" determina um risco cardiovascular intermediário entre normotensão e hipertensão, e a hipertensão mascarada, um risco semelhante à hipertensão (32). Embora ambas as condições sejam diagnosticadas com a realização da MAPA, esta ainda não faz parte da rotina de avaliação do paciente com DM. Os valores de referência de médias de PA na MAPA são: em 24 horas $\leq 135 / 80 \mathrm{mmHg}$, durante o dia $\leq 140 / 90$ $\mathrm{mmHg}$ e durante à noite $\leq 125 / 75 \mathrm{mmHg}(16,32)$.

A avaliação do perfil lipídico com medida de colesterol total, HDL-colesterol, triglicerídeos e estimativa do LDL-colesterol por meio da fórmula de Friedwald [colesterol total - (triglicerídeos/5+HDL); usar somente para valores de triglicerídeos $<400 \mathrm{mg} / \mathrm{dL}$ ] faz parte da estratificação de risco cardiovascular e do acompanhamento do paciente com DM e macroangiopatia. A estimativa do colesterol não HDL (colesterol total - HDL) pode ser também utilizada para avaliação do risco cardiovascular (16).

Tendo em vista a associação de maior risco cardiovascular em pacientes diabéticos com nefropatia e/ou insuficiência renal $(16,33)$, a avaliação da função renal por estimativa da taxa de filtração glomerular (TFG) por meio de fórmulas (MDRD: http://www.kidney. org/klsprofessionals/gfr_calculator.cfm) e pela medida de albuminúria em amostra casual de urina $(34,35)$ são indicadas na avaliação da macroangiopatia (36). Consideram-se como valores de referência: TFG $\geq 90$ $\mathrm{mL} / \mathrm{min}$ e albuminúria em amostra isolada $<14 \mathrm{mg} / \mathrm{L}$ (35). Deve ser realizada uma avaliação mínima de neuropatia autonômica com pesquisa de sintomas clássicos (tontura ao levantar-se, plenitude pós-prandial de longa duração, episódios de diarreia noturna, sudorese gustatória, impotência sexual) e da presença de hipotensão postural (16).
Na presença de aterosclerose coronariana importante, ou quando o risco cardiovascular estimado for elevado, recomenda-se que também sejam avaliados outros locais frequentes de doença macrovascular utilizando métodos específicos. A realização de ecografia de carótidas no paciente de alto risco cardiovascular tem sido recomendada por alguns autores, entretanto, sua indicação em pacientes assintomáticos ainda não está definida. Já a estenose crítica da artéria renal (> 70\%) ocorre em aproximadamente $17 \%$ dos pacientes DMT2 hipertensos, podendo ser associada à insuficiência renal e ao pior prognóstico cardiovascular. A redução da filtração glomerular ou aumento da creatinina sérica em mais de $30 \%$ associada ao uso de medicamentos inibidores da enzima conversora da angiotensina (iECA) ou bloqueadores dos receptores da angiotensina II (BRA) são indicações de avaliação de estenose de artéria renal, assim como hipertensão arterial resistente, episódios de edema agudo de pulmão, presença de sopro abdominal epigástrico e lateralizado. A ressonância magnética com angiografia é o método de escolha para essa avaliação, porém não deve ser utilizada em pacientes com TFG $<30 \mathrm{~mL} / \mathrm{min} / 1,73 \mathrm{~m}^{2}$ e pode ser usada com restrições para pacientes com TFG $<60$ $\mathrm{mL} / \mathrm{min} / 1,73 \mathrm{~m}^{2}$ por causa do risco do desenvolvimento de fibrose nefrogênica sistêmica (37). Por ocasião de estudos invasivos da circulação coronariana, pode-se fazer também avaliação simultânea das artérias renais. Quanto à DVP, não existem estudos avaliando se o rastreamento pela medida do índice pressórico tornozelo-braço reduz a morbimortalidade no $\mathrm{DM}$, não sendo recomendada sua realização no paciente assintomático.

\section{Avaliação da DAC}

O diagnóstico de cardiopatia isquêmica em pacientes diabéticos com sintomas típicos de angina pectoris segue as etapas habitualmente empregadas em pacientes sem DM, sendo essencialmente clínico. Deve ser lembrado que a presença de sintomas compatíveis com angina pectoris ou com IAM determina um risco relativo para eventos cardiovasculares de mais de duas vezes comparado à sua ausência (38). Em caso de dor típica, recomenda-se a estratificação de risco cardiovascular com testes funcionais (cintilografia miocárdica e/ou ecocardiograma de estresse). Já qualquer outro tipo de dor torácica, diferente dos pacientes sem DM, independente do sexo, indica realização de eletrocardiograma (ECG) de esforço (39). Embora o ECG de esforço tenha menor sensibilidade do que a cintilografia miocárdica e o ecocardiograma de estresse, por ter menor custo e trazer resultados quanto 
à estratificação de risco cardiovascular, é a primeira opção de teste funcional $(39,40)$. A cintilografia miocárdica e o ecocardiograma de estresse são indicados inicialmente para diagnóstico se o paciente for incapaz de realizar o ECG de esforço por limitações físicas ou na presença de alterações no ECG de repouso que possam confundir sua interpretação (síndrome de pré-excitação, uso de marcapasso, depressão ST > $1 \mathrm{~mm}$, bloqueio de ramo esquerdo ou fibrilação atrial) (41).

Uma vez feito o diagnóstico de DAC, a realização de cintilografia miocárdica ou ECG de esforço podem ser indicados para avaliar a gravidade da isquemia e a função ventricular. Cerca de $50 \%$ dos pacientes com DM não têm condições de realizar o esforço físico nestes testes (42), que também não estão indicados na presença de bloqueio de ramo esquerdo ou uso de marcapasso. Nestas situações, deverão ser utilizados testes funcionais com estresse farmacológico, pois sua acurácia é melhor por apresentar menos testes falsospositivos. Os resultados desses exames podem indicar mudanças na intensidade do tratamento antianginoso e/ou a realização de cineangiocoronariografia quando houver isquemia grave (39). Define-se isquemia grave como presença de isquemia reversível em mais de $20 \%$ da área do ventrículo esquerdo, em mais do que em um território suprido por artéria coronária, em múltiplos segmentos ou na presença de dilatação de cavidade ventricular esquerda. Deve ser lembrado que a cineangiocoronariografia é o teste de referência para o diagnóstico definitivo de DAC, mas, por ser invasivo e não isento de riscos (sangramento; 0,2\% de mortalidade), deve ser realizada apenas quando a perspectiva de procedimento de revascularização for uma possibilidade real (43). Angiografia coronária não invasiva pode ser realizada por meio de tomografia computadorizada ou ressonância magnética. Deve ser lembrado que esse exame apresenta menor acurácia diagnóstica do que a cineangiocoronariografia e não é recomendado para rastreamento de DAC, mesmo em pacientes de alto risco (44).

$\mathrm{Na}$ suspeita de síndrome coronariana aguda, o paciente deve ser encaminhado para uma emergência; confirmado o diagnóstico, será investigado com teste funcional e, dependendo do resultado deste último, por cineangiocoronariografia.

Métodos de imagem do processo aterosclerótico podem ajudar a estratificar o risco dos pacientes, uma vez que a presença de um processo aterosclerótico já é um indicativo do desenvolvimento de eventos cardiovasculares futuros. Além disso, permitem uma visão do impacto dos possíveis fatores de risco nas artérias do paciente avaliado. Os métodos de imagem mais utilizados incluem a medida do espessamento da camada íntimamédia da carótida por meio da ecografia com efeito Doppler e a medida do índice de calcificação coronariana pela tomografia computadorizada. Esses métodos são úteis especialmente nos pacientes com risco cardiovascular intermediário. A ausência de cálcio coronário torna pouco provável a presença de aterosclerose coronariana, enquanto sua presença/quantidade se associa à gravidade do processo aterosclerótico e desenvolvimento de eventos cardiovasculares. Pacientes com escore de cálcio $\geq 400$ apresentam uma taxa elevada de eventos cardiovasculares ( $\geq 20 \% \mathrm{em} \mathrm{dez}$ anos) (45). Mais recentemente, a disponibilidade de ultrassom intravascular pôde confirmar que os pacientes com DM têm placas ateroscleróticas maiores e mais vulneráveis do que aquelas de pacientes não diabéticos, achado este capaz de prever eventos cardiovasculares em longo prazo (46).

$\mathrm{O}$ aspecto que vem sendo debatido intensamente é a validade de realizar rastreamento para a presença de DAC em pacientes com DM sem sintomas de cardiopatia isquêmica, pois o DM já é considerado um equivalente à presença de DAC prévia. Nos pacientes com DM, as medidas intensivas de prevenção secundária, como controle rigoroso da PA (27) e uso de estatinas (47), já devem ser instituídas a partir do diagnóstico de DM.

A American Diabetes Association ( $\mathrm{ADA}$ ) não recomenda o rastreamento da DAC apenas com base na presença de outros fatores de risco cardiovasculares (16,42). A American Heart Association (AHA, 2007) recomenda o rastreamento apenas ao iniciar um programa de exercícios vigorosos.

O rastreamento da cardiopatia isquêmica assintomática no paciente com DM se justificaria por sua prevalência elevada, pela ausência de manifestações clínicas de DAC ou presença de manifestações atípicas em uma grande proporção de pacientes $(9,43)$. Além disso, nem todo paciente diabético deve ser considerado como um portador a priori de DAC (equivalente à presença de DAC). Idealmente, apenas aqueles com maior risco para desenvolver eventos cardiovasculares deveriam realizar o rastreamento. Uma sugestão é a de que a realização de testes diagnósticos para DAC não seja universal, mas baseada em um cálculo do risco cardiovascular (19), utilizando, por exemplo, o UKPDS risk engine. Devem ser levados em conta, também, outros aspectos não incluídos no cálculo de risco, como a história familiar de DCV precoce, história de eventos cardiovasculares 
prévios - incluindo amputações em membros inferiores, presença de doença renal e/ou neuropatia autonômica e sopro nas carótidas. Independente do risco estimado, todo o paciente com DM deve realizar ECG de repouso. $\mathrm{O}$ achado de ondas $\mathrm{Q}$, sinais que indiquem hipertrofia de ventrículo esquerdo, alterações do segmento ST e bloqueios de ramo indicam possível DAC e recomendase que seja realizada investigação suplementar.

A avaliação cardiovascular sugerida para os pacientes com DM assintomáticos para DAC está resumida na figura l. Conforme a estratificação de risco cardiovascular inicial, o paciente é classificado em baixo $(<10 \%$ de risco para evento cardiovascular em dez anos), médio ( $10 \%$ a $20 \%$ de risco para evento cardiovascular em dez anos) ou alto risco cardiovascular ( $>20 \%$ de risco para evento cardiovascular em dez anos) (17). Pacientes diabéticos com baixo risco cardiovascular podem ser reavaliados em 12 meses. Pacientes com risco cardiovascular intermediário e alto devem realizar pesquisa de cálcio coronário, não sendo indicada nenhuma investigação adicional se o escore de cálcio for $<400$. Escore de cálcio $>400$ indica teste funcional adicional (ECG esforço, respeitadas as limitações do teste ou cintilografia miocárdica ou ecocardiograma de estresse). Se o ECG de esforço sugerir isquemia miocárdica, a conduta subsequente deve ser individualizada (cintilografia miocárdica, cineangiocoronariografia), uma vez que o manejo desses pacientes não está ainda estabelecido de forma definitiva. Independente do risco cardiovascular calculado, a investigação adicional pode ser indicada quando o paciente apresentar algum dos seguintes fatores: alterações no ECG de repouso, história e/ou presença de DVP, AVC, neuropatia autonômica, micro/macroalbuminúria ou insuficiência renal.

\section{PREVENÇÃO E TRATAMENTO}

A tabela 2 descreve as principais intervenções na prevenção e no tratamento para o paciente com DM portador de macroangiopatia (DAC, AVC e DVP).

\section{Obesidade}

A perda de peso é recomendada nos pacientes com sobrepeso ou obesidade, por meio de alteração do estilo de vida, como redução da ingestão calórica associada ao aumento na atividade física. $\mathrm{O}$ uso de fármacos antiobesidade, quando indicados, pode ser mais efetivo na redução de peso (16). A redução da ingestão de gordura saturada, gorduras trans e colesterol é sempre recomendada.

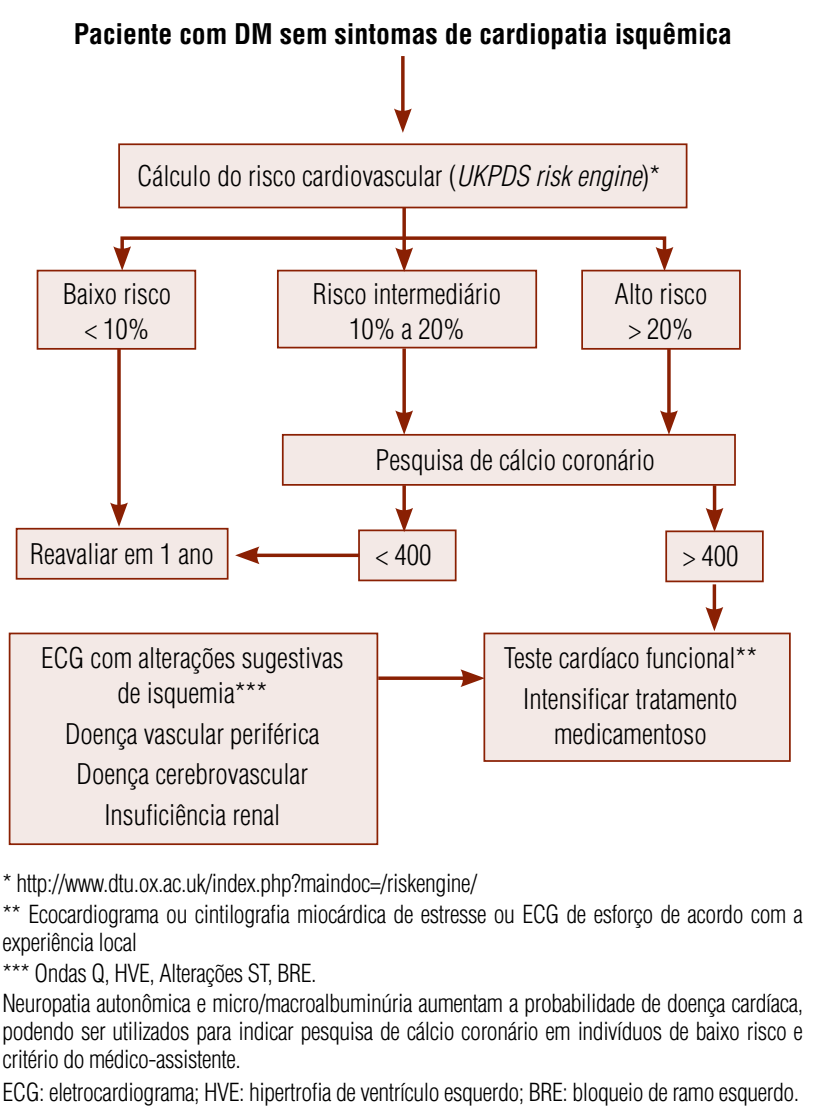

Figura 1. Algoritmo de avaliação cardiovascular sugerido para pacientes com DM sem sintomas de cardiopatia isquêmica.

\section{Sedentarismo}

Exercícios físicos melhoram o controle glicêmico no paciente com DMT2, contribuem para a perda de peso e reduzem a PA (48). Recomendam-se pelo menos $150 \mathrm{mi}-$ nutos de exercício aeróbico de intensidade moderada ( $50 \%$ a $70 \%$ da frequência cardíaca máxima) por semana ou pelo menos 90 minutos de exercício intenso por semana (16). Antes do início de prática de exercícios, o paciente deve realizar a avaliação da presença de doença macrovascular (DAC, DVP, AVC) e microvascular (16). Para pacientes com sequelas decorrentes de AVC prévio, deve ser adotado um programa de exercício supervisionado.

\section{Controle glicêmico}

Recomenda-se obter o melhor controle glicêmico possível, procurando-se atingir valores de $\mathrm{HbAlc}<7 \%$. É possível que o efeito do melhor controle glicêmico perdure mesmo após a interrupção deste, como sugerido pelo estudo EDIC (25). Esse estudo observacional acompanhou por nove anos a coorte original do DCCT após seu término. Foi demonstrada uma redução de $42 \%$ no desenvolvimento de desfechos cardiovasculares no grupo de pacientes que originalmente obteve um melhor 


\begin{tabular}{|c|c|c|}
\hline Condições & Medidas de intervenção & Metas a serem atingidas \\
\hline Obesidade & $\begin{array}{l}\text { Redução da ingestão calórica } \\
\text { Aumento na atividade física } \\
\text { Fármacos antiobesidade }\end{array}$ & $\mathrm{IMC}<25 \mathrm{~kg} / \mathrm{m}^{2}$ \\
\hline Sedentarismo & $\begin{array}{l}\text { Estimular aumento de atividade física } \\
\text { Supervisão de exercício, se sequelas de macroangiopatia }\end{array}$ & $\begin{array}{l}\text { Mínimo por semana: } \\
150 \text { minutos de exercício aeróbico com } 50 \% \text { a } 70 \% \text { da frequência } \\
\text { cardíaca máxima ou } 90 \text { minutos de exercício intenso }\end{array}$ \\
\hline Controle glicêmico & $\begin{array}{l}\text { Dieta hipocalórica } \\
\text { Aumento de atividade física } \\
\text { Agentes antidiabéticos orais } \\
\text { Insulina }\end{array}$ & $\begin{array}{l}\text { HbA1c }<7 \% \\
\text { Glicemia de jejum 70-130 mg/dL } \\
\text { Glicemia pós-prandial }<180 \text { mg/dL }\end{array}$ \\
\hline Controle pressórico & $\begin{array}{l}\text { Dieta DASH } \\
\text { Atividade física } \\
\text { Anti-hipertensivos: } \\
\text { iECA, ARAll, diuréticos, outros }\end{array}$ & $\begin{array}{l}<130 / 80 \mathrm{mmHg} \\
<125 / 75 \mathrm{mmHg} \text { (se } \uparrow \text { creatinina sérica e/ou proteinúria > 1,0 g/24 h) }\end{array}$ \\
\hline Dislipidemia & $\begin{array}{l}\text { Dieta para dislipidemia } \\
\text { Aumento de atividade física } \\
\text { Estatinas, fibratos, niacina } \\
\text { Controle glicêmico }\end{array}$ & $\begin{array}{l}\text { LDL-colesterol } \leq 100 \mathrm{mg} / \mathrm{dL} \text { ( } \leq 70 \mathrm{mg} / \mathrm{dL} \text { se múltiplos fatores de } \\
\text { risco ou tabagismo) } \\
\text { Triglicerídeos } \leq 150 \mathrm{mg} / \mathrm{dL} \\
\text { HDL-colesterol } \geq 40 \mathrm{mg} / \mathrm{dL} \text { não } \mathrm{HDL}<130 \mathrm{mg} / \mathrm{dL}\end{array}$ \\
\hline Estado pró-trombótico & $\begin{array}{l}\text { Aspirina } 75 \text { a } 162 \text { mg/dia: } \\
\text { - } \quad \text { prevenção secundária } \\
\text { - } \quad \text { prevenção primária, se risco cardiovascular elevado } \\
\text { Clopidogrel } 75 \text { mg/dia, se presença de doença vascular periférica }\end{array}$ & - \\
\hline Tabagismo & $\begin{array}{l}\text { Terapia comportamental } \\
\text { Terapia farmacológica }\end{array}$ & Cessação do fumo \\
\hline
\end{tabular}

controle glicêmico associado ao tratamento intensificado do DM. Em pacientes com DMT2, as evidências de que um melhor controle glicêmico diminui os eventos CV são menos claras. No estudo observacional do UKPDS, houve associação entre a redução da HbAlc e os eventos cardiovasculares, sem haver limiar de controle glicêmico. Para cada diminuição de 1 ponto percentual da HbAlc, houve redução de $18 \%$ no risco de desenvolver eventos cardiovasculares. Além disso, o seguimento da coorte original do UKPDS por mais dez anos demonstrou redução de $15 \%$ para a ocorrência de IAM (26). No último ano, foram publicados os resultados de três ensaios clínicos - ADVANCE (49), ACCORD (50), VADT (51) - que analisaram o efeito da obtenção de um controle glicêmico mais intensivo (HbAlc 6\%) em comparação às metas recomendadas $(\mathrm{HbAlc}<7 \%)$.

De maneira geral, a obtenção de controle glicêmico mais intensivo não foi mais benéfica em relação à redução de eventos cardiovasculares. No entanto, o tratamento intensivo reduziu eventos cardiovasculares em subgrupos de pacientes com níveis basais de $\mathrm{HbAlc}$ $<8 \%$ e sem história prévia de DCV (50), ou evidências de aterosclerose pelo escore de cálcio coronário (51) ou ainda em pacientes com menor duração do DM
(49). Consenso recente recomenda meta de HbAlc $<7 \%$ na prevenção da macroangiopatia do DM (ADA: recomendação grau B; AHA: recomendação IIb), individualizando as metas caso a caso (52).

O melhor controle glicêmico pode ser alcançado por meio do emprego da mudança do estilo de vida e medicamentos anti-hiperglicêmicos. Geralmente, utiliza-se uma combinação de medicamentos anti-hiperglicêmicos, usando-se fármacos que tenham mecanismos de ação diferentes. Embora não existam evidências conclusivas sobre redução do risco de DCV com qualquer medicamento antidiabético, incluindo insulina, recente metanálise demonstrou que a metformina foi o único agente que ofereceu redução no risco de mortalidade cardiovascular (53). Entretanto, qualquer medicamento antidiabético oral pode ser utilizado, desde que respeitadas as contraindicações e os cuidados específicos de cada classe.

A metformina deve ser suspensa temporariamente quando o paciente for hospitalizado por síndrome coronariana aguda, insuficiência cardíaca descompensada ou na insuficiência renal (TFG $<30 \mathrm{~mL} / \mathrm{min})(54)$. Em caso de realização de exames que necessitem o uso de contrastes endovenosos (cineangiocoronariografia, tomografia computadorizada), a metformina deve ser 
suspensa na noite anterior ao procedimento, pelo risco aumentado de acidose lática caso ocorra piora da função renal por nefropatia do contraste (55). O uso das glitazonas está contraindicado em pacientes com insuficiência cardíaca classes III e IV e/ou em associação com insulina em pacientes com cardiopatia estabelecida (56).

\section{Hipertensão arterial sistêmica}

Os pacientes com PA sistólica entre 130 e 139 mmHg ou diastólica entre 80 e $89 \mathrm{mmHg}$ devem realizar mudança do estilo de vida, com dieta e exercícios como tratamento único por, no máximo, três meses. A dieta inclui redução da ingestão de sódio e do excesso de calorias, aumento do consumo de frutas, vegetais e derivados do leite com pouca gordura e evitar o consumo excessivo de álcool (Dieta DASH, Dietary Approaches to Stop Hypertension). O alvo de PA é de < 130/80 $\mathrm{mmHg}(<125 / 75 \mathrm{mmHg}$ se creatinina elevada e proteinúria $>1,0 \mathrm{~g} / 24 \mathrm{~h}$ ). Caso os alvos de PA não sejam atingidos, deve ser iniciado tratamento farmacológico. No caso de PA sistólica $\geq 140 \mathrm{mmHg}$ ou diastólica $\geq 90$ $\mathrm{mmHg}$, o tratamento farmacológico deve ser concomitante à alteração do estilo de vida (16) e deve incluir um agente iECA ou antagonista do receptor da angiotensina II. Se necessário, um diurético deve ser adicionado (tiazídico ou diurético de alça, se a TFG for menor do que $30 \mathrm{~mL} / \mathrm{min}$, ou creatinina entre 2,5 e $3,0 \mathrm{mg} / \mathrm{dL}$ ) (29). Se iECA, antagonista do receptor da angiotensina II ou diurético forem usados, deve-se monitorizar o potássio sérico. A maioria dos pacientes necessitará de três ou mais drogas para atingir a PA alvo.

\section{Dislipidemia}

O uso de estatinas em pacientes diabéticos com DAC, com ou sem dislipidemia, reduz a mortalidade cardiovascular. Em pacientes sem DAC prévia, foi demonstrado que a redução de risco cardiovascular é obtida com o uso de estatinas naqueles pacientes com mais de 40 anos que tenham, além do DM, fatores de risco cardiovasculares associados (47).

$\mathrm{Na}$ presença de dislipidemia, a redução do LDL-colesterol é a principal meta do tratamento. Os valores de lipídeos séricos a serem atingidos com o tratamento da dislipidemia em pacientes com DM são: LDL-colesterol $\leq 100 \mathrm{mg} / \mathrm{dL}$, triglicerídeos $\leq 150 \mathrm{mg} / \mathrm{dL}$, HDL-colesterol $\geq 40 \mathrm{mg} / \mathrm{dL}$ e colesterol não $\mathrm{HDL}<130 \mathrm{mg} / \mathrm{dL}$. Na presença de risco cardiovascular elevado, recomendase como meta um valor de LDL-colesterol $\leq 70 \mathrm{mg} / \mathrm{dL}$. $\mathrm{O}$ tratamento com estatinas deve ser instituído associado à dieta nos pacientes que não atingirem níveis de LDLcolesterol alvo (16). Na presença de HDL-colesterol baixo e/ou hipertrigliceridemia, a terapia combinada com estatinas e niacina ou estatinas e fibrato pode ser utilizada (16). No entanto, essa conduta não foi avaliada quanto a desfechos cardiovasculares ou segurança em ensaios clínicos, havendo, além disso, maior risco para elevação das transaminases, miosite e rabdomiólise.

\section{Prevenção de trombose}

A aspirina deve ser usada na dose de 75 a $162 \mathrm{mg} /$ dia na prevenção secundária de pacientes diabéticos com história de DCV estabelecida (57). O uso de aspirina como prevenção primária tem sido indicado nos pacientes diabéticos com risco cardiovascular aumentado, isto é, aqueles com mais de 40 anos de idade ou com fator de risco cardiovascular adicional (história familiar de DCV, hipertensão, tabagismo, dislipidemia ou microalbuminúria) (16). Entretanto, esse ainda é um tema controverso. A terapia combinada com clopidrogel pode ser usada em pacientes com DCV grave e progressiva, além de ser uma alternativa para pacientes com contraindicação à aspirina (58). Já em pacientes com DVP e DM, o clopidogrel, $75 \mathrm{mg} /$ dia, é mais efetivo do que a aspirina para reduzir eventos cardiovasculares (59).

\section{Tabagismo}

Todos os pacientes diabéticos devem ser orientados a cessar o tabagismo por meio de terapia comportamental e/ou farmacológica (16).

\section{Doença arterial coronariana estabelecida}

A prescrição de pacientes com DAC deve incluir iECA (60), aspirina e estatinas (61) e, em caso de IAM prévio, um betabloqueador (62), com o objetivo de redução de mortalidade. $\mathrm{O}$ uso de outros medicamentos antianginosos deve ser individualizado (nitratos e bloqueadores do canal do cálcio).

As indicações de cirurgia são as mesmas utilizadas para os pacientes sem DM. Existem poucos estudos comparando o tratamento medicamentoso e cirúrgico em pacientes com DM e DAC. A cirurgia aberta é superior à angioplastia com stents, quando indicada revascularização miocárdica (63).

A presença de DM é um fator de risco independente de reestenose e eventos clínicos adversos em inúmeros estudos com stents, recobertos ou não $(4,10)$. Em pacientes com angina estável, com e sem DM, submetidos a um tratamento clínico ótimo, não há aumento de sobrevida 
com o emprego concomitante de stents coronarianos (64). Nesses estudos, não foram avaliados stents recobertos com drogas ou inibidores da glicoproteína IIa/IIb duas intervenções que melhoram o resultado da angioplastia em pacientes diabéticos. Estudo recente demonstrou uma menor taxa de necessidade de revascularização com a utilização de stents recobertos em pacientes com DM quando comparado a stents não recobertos. Deve ser salientado que os pacientes devem permanecer em uso de tratamento antiplaquetário duplo (aspirina e clopidogrel) por pelo menos seis meses após o procedimento (65). Mesmo considerando que os stents recobertos diminuem em cerca de $75 \%$ a reintervenção em pacientes com DM quando comparados a stents não recobertos, a reintervenção ainda é duas vezes mais frequente do que nos pacientes sem DM (10). Até o presente momento, na angina estável, parece que o tratamento clínico ótimo inicial (64) e, se necessária, a cirurgia aberta (63) são as melhores opções para os pacientes com DM e DAC. No paciente com angina instável, a revascularização percutânea pode ser uma opção, respeitando as mesmas indicações de pacientes não diabéticos.

Em relação ao paciente assintomático para DAC, a observação de que, após tratamento medicamentoso, $79 \%$ deles apresentam resolução de isquemia à cintilografia miocárdica (66) e que o rastreamento de indivíduos assintomáticos não muda seu prognóstico (67), sugere que o manejo clínico por meio de medicamentos deve ser a conduta indicada nesses pacientes. Entretanto, um estudo retrospectivo realizado em pacientes diabéticos assintomáticos demonstrou que a revascularização por cirurgia aberta determinou uma maior sobrevida em relação ao tratamento medicamentoso ou à angioplastia percutânea nos pacientes que tinham evidência de isquemia grave na cintilografia miocárdica (68). Uma análise dos resultados agrupados de dez estudos randomizados, que compararam a efetividade da revascularização miocárdica por cirurgia ou percutânea, concluiu que os pacientes diabéticos obtiveram redução da mortalidade de aproximadamente $30 \%$ após o procedimento cirúrgico (69).

\section{Acidente vascular cerebral estabelecido}

Pacientes que tiveram AVC isquêmico ou episódio isquêmico transitório devem eliminar o consumo de álcool. Aqueles que apresentam fibrilação atrial associada ao AVC devem ser anticoagulados com agentes orais, com o objetivo de um INR entre 2 e 3 . Havendo contraindicação de anticoagulação oral, deve ser utilizada a aspirina $(325 \mathrm{mg} / \mathrm{dia})(70)$. Na doença cerebrovascular, a endarterectomia está indicada quando houver estenose de carótida $\geq 70 \%$, pois esse procedimento reduz o risco de AVC quando comparado ao tratamento medicamentoso na mesma situação (71).

\section{Doença vascular periférica estabelecida}

Pacientes com DVP sintomática devem realizar exercícios físicos supervisionados (caminhada em esteira pelo menos três vezes/semana) (72). O cilostazol pode ser utilizado em pacientes sem resposta aos exercícios, não devendo ser utilizado nos pacientes com insuficiência cardíaca. Além disso, todos os pacientes com DVP devem ser orientados quanto à prevenção de problemas relacionados ao pé diabético e à dor em repouso. A ulceração ou a gangrena do pé devem ser tratadas de imediato (16). A revascularização cirúrgica (cirurgia aberta ou endovascular) deve ser indicada na presença de claudicação incapacitante ou isquemia crítica, representada por dor em repouso e/ou lesão trófica. Grandes amputações são indicadas quando houver infecção com ameaça à vida do paciente, dor em repouso não controlada ou necrose extensa por oclusão de artéria principal (14).

Deve ser lembrado que uma intervenção multifatorial intensiva que inclua mudança no estilo de vida, controle glicêmico intensivo, uso de iECA, aspirina, estatinas e abordagem com equipe multidisciplinar é capaz de reduzir o risco de DCV fatal em pacientes com DMT2 e microalbuminúria (73).

\section{CONSIDERAÇÕES FINAIS}

Além de maior risco para macroangiopatia, pacientes com DM e DCV têm pior prognóstico, apresentam menor sobrevida em curto prazo, maior risco de recorrência da doença e pior resposta aos tratamentos. Os avanços diagnósticos e terapêuticos das últimas décadas já mostram seus primeiros resultados, com redução de $50 \%$ do risco de eventos cardiovasculares nestes pacientes, mas o risco absoluto de eventos é ainda duas vezes maior em relação ao dos pacientes não diabéticos. Com base nas evidências apresentadas neste estudo, recomenda-se a adoção de uma estratégia de manejo agressivo, na qual seja prioritário o controle rígido dos fatores de risco cardiovasculares.

Declaração: o autor JLG participou dos conselhos consultivos da Bristol-Myers Squibb, Eli Lilly, Glaxo SmithKline, Novo Nordisk, Sanofi Aventis, Condução de estudos clínicos: Abbott, Bristol-Myers Squibb, Eli Lilly, Glaxo SmithKline, MannKind Co., Merck Sharp \& Dohme, Novo Nordisk, Roche, Sanofi Aventis. 


\section{REFERÊNCIAS}

1. Scheffel RS, Bortolanza D, Weber CS, Costa LA, Canani LH, Santos $\mathrm{KG}$, et al. Prevalence of micro and macroangiopatic chronic complications and their risk factors in the care of out patients with type 2 diabetes mellitus. Rev Assoc Med Bras. 2004;50(3):263-7.

2. Schaan $B D$, Harzheim E, Gus I. Cardiac risk profile in diabetes mellitus and impaired fasting glucose. Rev Saude Publica. 2004;38(4):529-36.

3. Quadros AS, Sarmento-Leite R, Bertoluci M, Duro K, Schmidt A, De Lucca $G$ Jr., et al. Angiographic coronary artery disease is associated with progressively higher levels of fasting plasma glucose. Diabetes Res Clin Pract. 2007;75(2):207-13.

4. Schaan BD, Quadros A, Sarmento-Leite R, Gottschall CA. Diabetes and coronary stent implantation: experience from a reference center in interventional cardiology. Arq Bras Endocrinol Metabol. 2006;50(1):38-45.

5. Borch-Johnsen K, Kreiner S. Proteinuria: value as predictor of cardiovascular mortality in insulin dependent diabetes mellitus. Br Med J (Clin Res Ed). 1987;294(6588):1651-4.

6. Stamler J, Vaccaro O, Neaton JD, Wentworth D. Diabetes, other risk factors, and 12-yr cardiovascular mortality for men screened in the Multiple Risk Factor Intervention Trial. Diabetes Care. 1993;16(2):434-44.

7. Stegmayr B, Asplund K. Diabetes as a risk factor for stroke. A population perspective. Diabetologia. 1995;38(9):1061-8.

8. Canavan RJ, Unwin NC, Kelly WF, Connolly VM. Diabetes- and nondiabetes-related lower extremity amputation incidence before and after the introduction of better organized diabetes foot care: continuous longitudinal monitoring using a standard method. Diabetes Care. 2008;31(3):459-63.

9. Beck MO, Silveiro SP, Friedman R, Clausell N, Gross JL. Asymptomatic coronary artery disease is associated with cardiac autonomic neuropathy and diabetic nephropathy in type 2 diabetic patients. Diabetes Care. 1999;22(10):1745-7.

10. Quadros A, Diemer F, Lima T, Abdalla R, Vizotto M, Gottschall CA, et al. Percutaneous coronary intervention in diabetes mellitus: an updated analysis of medical practice. Arq Bras Endocrinol Metabol. 2007;51(2):327-33.

11. Malmberg K, Ryden L, Hamsten A, Herlitz J, Waldenstrom A, Wedel $\mathrm{H}$. Effects of insulin treatment on cause-specific one-year mortality and morbidity in diabetic patients with acute myocardial infarction. DIGAMI Study Group. Diabetes Insulin-Glucose in Acute Myocardial Infarction. Eur Heart J. 1996;17(9):1337-44.

12. Tuomilehto J, Rastenyte D, Jousilahti P, Sarti C, Vartiainen E. Diabetes mellitus as a risk factor for death from stroke. Prospective study of the middle-aged finnish population. Stroke. 1996;27(2):210-5.

13. MeijerWT, Hoes AW, Rutgers D, Bots ML, Hofman A, Grobbee DE. Peripheral arterial disease in the elderly: The Rotterdam Study. Arterioscler Thromb Vasc Biol. 1998;18(2):185-92.

14. Peripheral arterial disease in people with diabetes. Diabetes Care. 2003;26(12):3333-41.

15. Pickett CA, Jackson JL, Hemann BA, Atwood JE. Carotid bruits as a prognostic indicator of cardiovascular death and myocardial infarction: a meta-analysis. Lancet. 2008;371(9624):1587-94.

16. Standards of medical care in diabetes - 2009. Diabetes Care. 2009;32 Suppl 1:S13-61.

17. Third Report of the National Cholesterol Education Program (NCEP) Expert Panel on Detection, Evaluation, and Treatment of High Blood Cholesterol in Adults (Adult Treatment Panel III) final report. Circulation. 2002;106(25):3143-421.

18. Evans JM, Wang J, Morris AD. Comparison of cardiovascular risk between patients with type 2 diabetes and those who had had a myocardial infarction: cross sectional and cohort studies. BMJ. 2002;324(7343):939-42.
19. Dora JM, Kramer CK, Canani LH. Standards of Medical Care in Diabetes - 2008: response to Hirsch, Inzucchi, and Kirkman. Diabetes Care 2008;31(5):e44; author reply e45.

20. Stevens RJ, Kothari V, Adler Al, Stratton IM. The UKPDS risk engine: a model for the risk of coronary heart disease in type II diabetes (UKPDS 56). Clin Sci (Lond). 2001;101(6):671-9.

21. Best LG, Zhang Y, Lee ET, Yeh JL, Cowan L, Palmieri V, et al. Creactive protein as a predictor of cardiovascular risk in a population with a high prevalence of diabetes: the Strong Heart Study. Circulation. 2005;112(9):1289-95.

22. Ridker PM, Danielson E, Fonseca FA, Genest J, Gotto AM Jr., Kastelein JJ, et al. Rosuvastatin to prevent vascular events in men and women with elevated C-reactive protein. N Engl J Med. 2008;359(21):2195-207.

23. Saremi A, Nelson RG, Tulloch-Reid M, Hanson RL, Sievers ML, Taylor GW, et al. Periodontal disease and mortality in type 2 diabetes. Diabetes Care. 2005;28(1):27-32.

24. Effect of intensive blood-glucose control with metformin on complications in overweight patients with type 2 diabetes (UKPDS 34). UK Prospective Diabetes Study (UKPDS) Group. Lancet. 1998;352(9131):854-65.

25. The Diabetes Control and Complications Trial/Epidemiology of Diabetes Interventions and Complications (DCCT/EDIC) Study Research Group. Intensive Diabetes treatment and cardiovascular disease in patients with type 1 diabetes. N Engl J Med. 2005;353(25):2643-53.

26. Holman RR, Paul SK, Bethel MA, Matthews DR, Neil HA. 10-year follow-up of intensive glucose control in type 2 diabetes. N Engl J Med. 2008;359(15):1577-89.

27. Tight blood pressure control and risk of macrovascular and microvascular complications in type 2 diabetes: UKPDS 38. UK Prospective Diabetes Study Group. BMJ. 1998;317(7160):703-13.

28. Holman RR, Paul SK, Bethel MA, Neil HA, Matthews DR. Longterm follow-up after tight control of blood pressure in type 2 diabetes. N Engl J Med. 2008;359(15):1565-76.

29. Chobanian AV, Bakris GL, Black HR, Cushman WC, Green LA, Izzo $\mathrm{JL}$ Jr., et al. Seventh report of the Joint National Committee on Prevention, Detection, Evaluation, and Treatment of High Blood Pressure. Hypertension. 2003;42(6):1206-52.

30. Kramer CK, Leitao CB, Canani LH, Gross JL. Impact of white-coat hypertension on microvascular complications in type 2 diabetes. Diabetes Care. 2008;31(12):2233-7.

31. Leitao CB, Canani LH, Kramer CK, Boza JC, Pinotti AF, Gross $\mathrm{JL}$. Masked hypertension, urinary albumin excretion rate, and echocardiographic parameters in putatively normotensive type 2 diabetic patients. Diabetes Care. 2007;30(5):1255-60.

32. Leitao CB, Canani LH, Silveiro SP, Gross JL. Ambulatory blood pressure monitoring and type 2 diabetes mellitus. Arq Bras Cardiol. 2007;89(5):315-21, 347-54.

33. Gross JL, Silveiro SP, Canani LH, Friedman R, Leitao CB, Azevedo MJ. Diabetic nephropathy and cardiac disease. Arq Bras Endocrinol Metabol. 2007;51(2):244-56.

34. Gross JL, de Azevedo MJ, Silveiro SP, Canani LH, Caramori ML, Zelmanovitz T. Diabetic nephropathy: diagnosis, prevention, and treatment. Diabetes Care. 2005;28(1):164-76.

35. Incerti J, Zelmanovitz T, Camargo JL, Gross JL, de Azevedo MJ. Evaluation of tests for microalbuminuria screening in patients with diabetes. Nephrol Dial Transplant. 2005;20(11):2402-7.

36. Gross ML, Dikow R, Ritz E. Diabetic nephropathy: recent insights into the pathophysiology and the progression of diabetic nephropathy. Kidney Int Suppl. 2005(94):S50-3.

37. Deo A, Fogel M, Cowper SE. Nephrogenic systemic fibrosis: a population study examining the relationship of disease development to gadolinium exposure. Clin J Am Soc Nephrol. 2007;2(2):264-7.

38. Azevedo MJ, Neto AF, Caramori ML, Beck MO, Moreira JS, Ludwig $R$, et al. Value of diagnostic tools for myocardial ischemia used in routine clinical practice to predict cardiac events in pa- 
tients with type 2 diabetes mellitus: a prospective study. Arq Bras Endocrinol Metabol. 2006;50(1):46-52.

39. Snow V, Barry P, Finn SD, Gibbons RJ, Owens DK, Williams SV, et al. Evaluation of primary care patients with chronic stable angina: guidelines from the American College of Physicians. Ann Intern Med. 2004;141(1):57-64.

40. Bax JJ, Young LH, Frye RL, Bonow RO, Steinberg HO, Barrett EJ. Screening for coronary artery disease in patients with diabetes. Diabetes Care. 2007;30(10):2729-36.

41. Bax JJ, Inzucchi SE, Bonow RO, Schuijf JD, Freeman MR, Barrett EJ. Cardiac imaging for risk stratification in diabetes. Diabetes Care. 2007;30(5):1295-304.

42. Wackers FJ, Young LH, Inzucchi SE, Chyun DA, Davey JA, Barrett EJ, et al. Detection of silent myocardial ischemia in asymptomatic diabetic subjects: the DIAD study. Diabetes Care. 2004;27(8):1954-61.

43. Snow V, Barry P, Finn SD, Gibbons RJ, Owens DK, Williams SV, et al. Primary care management of chronic stable angina and asymptomatic suspected or known coronary artery disease: a clinical practice guideline from the American College of Physicians. Ann Intern Med. 2004;141(7):562-7.

44. Miller JM, Rochitte CE, Dewey M, Arbab-Zadeh A, Niinuma $H$, Gottlieb I, et al. Diagnostic performance of coronary angiography by 64-row CT. N Engl J Med. 2008;359(22):2324-36.

45. Greenland $\mathrm{P}$, Bonow RO, Brundage BH, Budoff MJ, Eisenberg MJ, Grundy SM, et al. ACCF/AHA 2007 clinical expert consensus document on coronary artery calcium scoring by computed tomography in global cardiovascular risk assessment and in evaluation of patients with chest pain: a report of the American College of Cardiology Foundation Clinical Expert Consensus Task Force (ACCF/AHA Writing Committee to Update the 2000 Expert Consensus Document on Electron Beam Computed Tomography). Circulation. 2007;115(3):402-26.

46. Kim SH, Hong MK, Park DW, Lee SW, Kim YH, Lee CW, et al. Impact of plaque characteristics analyzed by intravascular ultrasound on long-term clinical outcomes. Am J Cardiol. 2009;103(9):1221-6.

47. Colhoun HM, Betteridge DJ, Durrington PN, Hitman GA, Neil HA, Livingstone SJ, et al. Primary prevention of cardiovascular disease with atorvastatin in type 2 diabetes in the Collaborative Atorvastatin Diabetes Study (CARDS): multicentre randomised placebo-controlled trial. Lancet. 2004;364(9435):685-96.

48. Boule NG, Haddad E, Kenny GP, Wells GA, Sigal RJ. Effects of exercise on glycemic control and body mass in type 2 diabetes mellitus: a meta-analysis of controlled clinical trials. JAMA. 2001;286(10):1218-27.

49. Patel A, MacMahon S, Chalmers J, Neal B, Billot L, Woodward M, et al. Intensive blood glucose control and vascular outcomes in patients with type 2 diabetes. N Engl J Med. 2008;358(24):2560-72.

50. Gerstein HC, Miller ME, Byington RP, Goff DC Jr., Bigger JT, Buse $\mathrm{JB}$, et al. Effects of intensive glucose lowering in type 2 diabetes. N Engl J Med. 2008;358(24):2545-59.

51. Duckworth W, Abraira C, Moritz T, Reda D, Emanuele N, Reaven PD, et al. Glucose control and vascular complications in veterans with type 2 diabetes. N Engl J Med. 2009;360(2):129-39.

52. Skyler JS, Bergenstal R, Bonow RO, Buse J, Deedwania P, Gale $E A$, et al. Intensive glycemic control and the prevention of cardiovascular events: implications of the ACCORD, ADVANCE, and VA diabetes trials. A Position Statement of the American Diabetes Association and a Scientific Statement of the American College of Cardiology Foundation and the American Heart Association. Circulation. 2008.

53. Selvin E, Bolen S, Yeh HC, Wiley C, Wilson LM, Marinopoulos SS, et al. Cardiovascular outcomes in trials of oral diabetes medications: a systematic review. Arch Intern Med. 2008;168(19):2070-80.

54. Nathan DM, Buse JB, Davidson MB, Ferrannini E, Holman RR, Sherwin $R$, et al. Medical management of hyperglycemia in type 2 diabetes: a consensus algorithm for the initiation and adjustment of therapy: a consensus statement of the American Diabe- tes Association and the European Association for the Study of Diabetes. Diabetes Care. 2009;32(1):193-203.

55. Gross JL, Friedman R, Silveiro SP. Preventing nephropathy induced by contrast medium. N Engl J Med. 2006;354(17):1853-5; author reply 1853-5.

56. Nesto RW, Bell D, Bonow RO, Fonseca V, Grundy SM, Horton ES, et al. Thiazolidinedione use, fluid retention, and congestive heart failure: a consensus statement from the American Heart Association and American Diabetes Association. Diabetes Care. 2004;27(1):256-63.

57. Colwell JA. Aspirin therapy in diabetes. Diabetes Care. 2004;27 Suppl 1:S72-3.

58. Bhatt DL, Marso SP, Hirsch AT, Ringleb PA, Hacke W, Topol EJ. Amplified benefit of clopidogrel versus aspirin in patients with diabetes mellitus. Am J Cardiol. 2002;90(6):625-8.

59. A randomised, blinded, trial of clopidogrel versus aspirin in patients at risk of ischaemic events (CAPRIE). CAPRIE Steering Committee. Lancet. 1996;348(9038):1329-39.

60. Effects of ramipril on cardiovascular and microvascular outcomes in people with diabetes mellitus: results of the HOPE study and MICRO-HOPE substudy. Heart Outcomes Prevention Evaluation Study Investigators. Lancet. 2000;355(9200):253-9.

61. Vijan S, Hayward RA. Pharmacologic lipid-lowering therapy in type 2 diabetes mellitus: background paper for the American College of Physicians. Ann Intern Med. 2004;140(8):650-8.

62. Malmberg K, Herlitz J, Hjalmarson A, Ryden L. Effects of metoprolol on mortality and late infarction in diabetics with suspected acute myocardial infarction. Retrospective data from two large studies. Eur Heart J. 1989;10(5):423-8.

63. Influence of diabetes on 5-year mortality and morbidity in a randomized trial comparing CABG and PTCA in patients with multivessel disease: the Bypass Angioplasty Revascularization Investigation (BARI). Circulation. 1997;96(6):1761-9.

64. Boden WE, O'Rourke RA, Teo KK, Hartigan PM, Maron DJ, Kostuk WJ, et al. Optimal medical therapy with or without PCl for stable coronary disease. N Engl J Med. 2007;356(15):1503-16.

65. Stettler C,Allemann S, Wandel S, Kastrati A, Morice MC, Schomig A, et al. Drug eluting and bare metal stents in people with and without diabetes: collaborative network meta-analysis. BMJ. 2008;337:a1331.

66. Wackers FJ, Chyun DA, Young LH, Heller GV, Iskandrian AE, Davey $\mathrm{JA}$, et al. Resolution of asymptomatic myocardial ischemia in patients with type 2 diabetes in the Detection of Ischemia in Asymptomatic Diabetics (DIAD) study. Diabetes Care. 2007;30(11):2892-8.

67. Young LH, Wackers FJ, Chyun DA, Davey JA, Barrett EJ, Taillefer R, et al. Cardiac outcomes after screening for asymptomatic coronary artery disease in patients with type 2 diabetes: the DIAD study: a randomized controlled trial. JAMA. 2009;301(15):1547-55.

68. Sorajja $\mathrm{P}$, Chareonthaitawee $\mathrm{P}$, Rajagopalan N, MillerTD, Frye RL, Hodge DO, et al. Improved survival in asymptomatic diabetic patients with high-risk SPECT imaging treated with coronary artery bypass grafting. Circulation. 2005;112(9 Suppl):I311-6.

69. Hlatky MA, Boothroyd DB, Bravata DM, Boersma E, Booth J, Brooks MM, et al. Coronary artery bypass surgery compared with percutaneous coronary interventions for multivessel disease: a collaborative analysis of individual patient data from ten randomised trials. Lancet. 2009;373(9670):1190-7.

70. Ezekowitz MD, Levine JA. Preventing stroke in patients with atrial fibrillation. JAMA. 1999;281(19):1830-5.

71. Barnett HJ, Taylor DW, Eliasziw M, Fox AJ, Ferguson GG, Haynes $\mathrm{RB}$, et al. Benefit of carotid endarterectomy in patients with symptomatic moderate or severe stenosis. North American Symptomatic Carotid Endarterectomy Trial Collaborators. N Engl J Med. 1998;339(20):1415-25.

72. Leng GC, Fowler B, Ernst E. Exercise for intermittent claudication. Cochrane Database of Systematic Reviews (Online : Update Software) 2000(2):CD000990.

73. Gaede $\mathrm{P}$, Lund-Andersen $\mathrm{H}$, Parving $\mathrm{HH}$, Pedersen O. Effect of a multifactorial intervention on mortality in type 2 diabetes. N Engl J Med. 2008;358(6):580-91. 